\title{
Plastic Flow Macrolocalization: Autowave and Quasi-Particle
}

\author{
Lev B. Zuev, Svetlana A. Barannikova \\ Institute of Strength Physics and Materials Science, Tomsk, Russia \\ E-mail:lbz@ispms.tsc.ru \\ Received March 2, 2010; revised March 26, 2010; accepted April 7, 2010
}

\begin{abstract}
A new approach is proposed to describe the autowave processes responsible for plastic deformation localization in metals and alloys. The existence of a quasi-particle, which corresponds to a localized plastic flow autowave, is postulated and its characteristics are determined. The above postulate leads to a number of corollaries and quantitative assessments that are considered herein. The deformation processes occurring on the macro- and micro-scale levels are found to be directly related.
\end{abstract}

Keywords: Localization, Plastic Deformation, Autowave, Quasi-Particle

\section{Introduction}

The experimental studies of plastic flow in solids carried out during last decades $[1,2]$ allow one to throw light upon the plasticity phenomenon and to detect the most important experimental fact that the plastic flow would exhibit a localization behavior from yield point to failure. Recently strong experimental evidences for the above viewpoint were presented independently by several workers [3-5]. In order to visualize the localization patterns observed by mechanical testing ${ }_{2}$ a technique of double-exposure speckle-photography was developed [6].

Some of the observed localization patterns are demonstrated in Figure 1(a). As is seen from Figure 1(b), these have very complex structure characterized by a typical macroscopic scale of about $10^{-2} \mathrm{~m}$. This suggests that the deforming medium becomes spontaneously stratified into macroscopic layers, with deforming (active) layers alternating with non-deforming (passive) ones. In a general case, the boundaries between such layers are mobile; therefore, the process of plastic flow is conventionally considered as evolution of localized plastic flow patterns.

The phenomenology and quantitative characteristics of the localization effect have been fully elucidated by now. Thus a detailed investigation of space-time periodic localization patterns $[1,2]$ allows one to refer the localization phenomenon to self-organization processes. The above assumption is only valid provided the term 'selforganization' is taken to imply, according to Haken [7], that the system acquires spatial, temporal or functional structure in the absence of any specific periodic external action. Of major importance is the finding that the localized plastic flow patterns have all the particular features of autowave (self-excited) process. This comes into particular prominence at the linear stage of deformation hardening as the plastic flow localization takes on the form of phase autowave, which has length $\lambda \approx 10^{-2} \mathrm{~m}$ and propagation rate $10^{-5} \leq V_{a w} \leq 10^{-4} \mathrm{~m} \cdot \mathrm{s}^{-1}$. The autowaves in question are distinct from the well-known plastic deformation waves that are generated in solids under shock loading which are described by Kolsky in [8].

However, the nature of localized plastic flow phenomena is poorly understood so that the challenge of interpreting rich experimental evidences on plastic flow macrolocalization can be daunting. To accomplish this demanding task, a new model of plastic flow localization is proposed herein.

\section{On the Observation of Localization Phenomena}

As noted above, the experimental observation of localized plastic flow autowaves was carried on with help of a specially developed speckle photography technique related to focused-image holography [6]. The method developed makes feasible the experimental determination of displacement vector fields and the calculation of plastic distortion tensor components for the deforming specimen. A vast array of wavelength and propagation rate data has been acquired and stored digitally in a computer. 


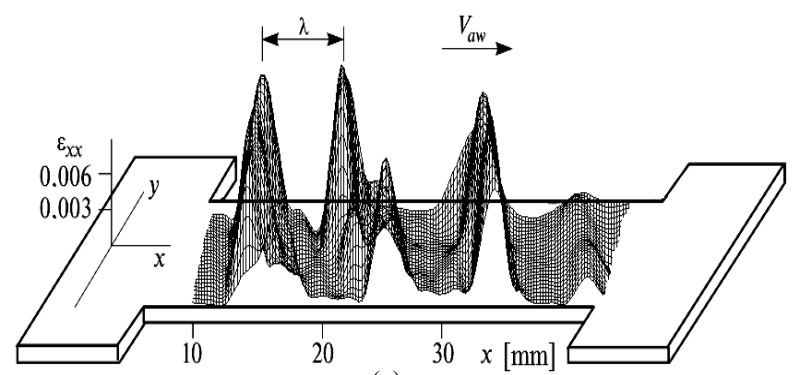

(a)

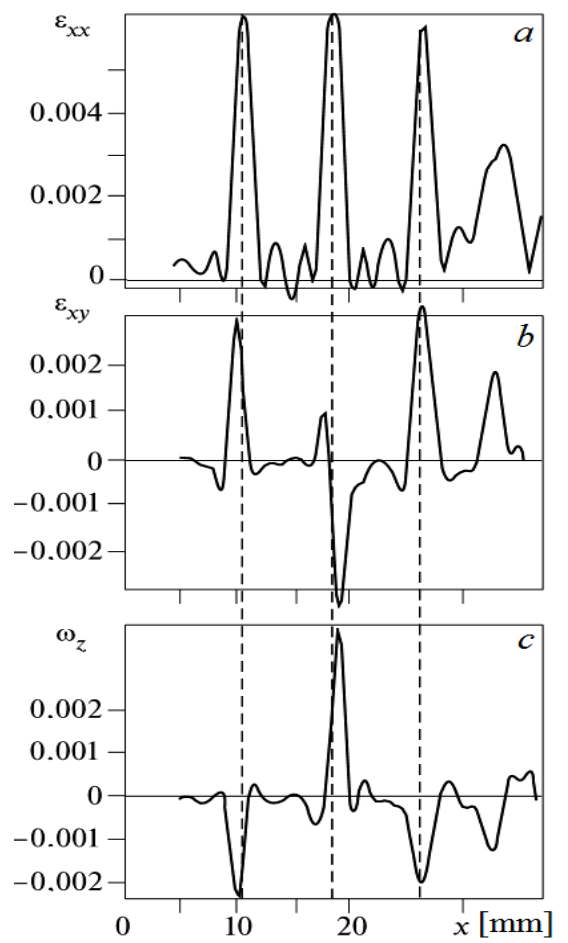

(b)

Figure 1. (a) A typical example of localized plastic flow autowave generated at the linear work hardening stage in the single crystal of alloyed $\boldsymbol{\gamma}$ - Fe; (b) $\varepsilon_{x x}$ - local elongation; $\boldsymbol{x}$ and $y$-specimen length and width, respectively; $\lambda$ - nucleus spacing (autowave length); $V_{a w}$ - autowave propagation rate; the distributions of the plastic distortion tensor components, $\varepsilon_{x x}, \varepsilon_{x y}$ and $\omega_{z}$, within the localization zone in the single crystal of alloyed $\gamma$-Fe.

The spatial distributions of plastic distortion tensor components can be used to locate localized plastic flow nuclei; the kinetic characteristics of the nuclei can be determined from the temporal evolution thereof. The characteristics of autowaves are defined as follows. First the spatial period (length of autowave, $\lambda$ ) is determined from the variation in the space co-ordinates of localization nuclei with time (see Figure 2); then the time of variation, $T$, is defined. Hence the phase rate of autowave propagation is given as $V_{a w}=\lambda / T=\omega / k$ (here $\omega=2 \pi / T$ is the frequency and $k=2 \pi / \lambda$ is the wave number).

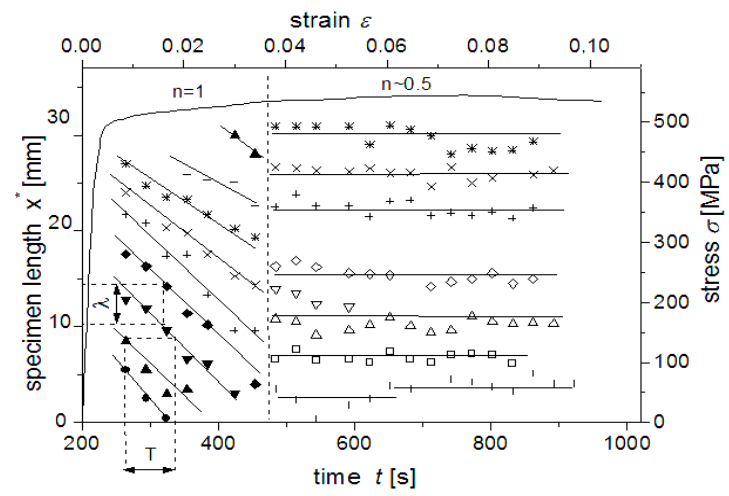

Figure 2. The spatial $(\lambda)$ and temporal $(T)$ periods of localized plastic deformation as determined for the single crystals of alloyed $\gamma$-Fe for $n=1$ and $n \sim 0.5$ (linear and parabolic work hardening stages, respectively); $\sigma(\varepsilon)$ - stressstrain dependence; $X(t)$ - variation in the localization nucleus co-ordinates with time $(\bullet ; \Delta ; \nabla ; \square ;+; \times ; * ;-)$.

The quantitative characteristics of autowave processes involved in the plastic flow localization were determined experimentally in our investigations [1,2]. The test specimens were prepared from the single crystals of FCC BCC and HCP alloys (Al, $\mathrm{Cu}, \mathrm{Ni}$ and $\gamma$-Fe; $\gamma$-Fe, $\mathrm{V}$ and $\mathrm{Nb}$ and $\mathrm{Mg}, \mathrm{Zn}, \mathrm{Zr}$ and $\mathrm{Ti}$, respectively) and from polycrystalline metals and alloys. The alkali halide crystals $\mathrm{KCl}, \mathrm{NaCl}$ and $\mathrm{LiF}$ and some rocks were also studied. The mechanical characteristics and the shape of plastic flow curve are found to be determined by chemical composition, grain size (in the case of polycrystals) and tension axis orientation (in the case of single crystals). The plastic localization patterns observed for all materials studied have many features in common which are discussed below.

\section{Correspondence between the Localized Plastic Flow Patterns and the Work Hardening Stages}

One of the striking results obtained in these investigations is the finding that the emergent localized plastic flow patterns strictly correspond to the well-known plastic strain stages [9].

Using the Ludwik equation [10], the flow curve $\sigma(\varepsilon)$ is readily separated into individual stages, i.e.

$$
\sigma(\varepsilon)=\sigma_{0}+\theta \varepsilon^{n},
$$

where $\sigma_{0}$ is the proof stress, $\theta$ is the work hardening coefficient and $n$ is the hardening exponent. Especially convenient for this separation is the value $n$ since it changes discretely with the plastic flow. Then the flow stages singled out on the curve $\sigma(\varepsilon)$ are matched against the respective specific patterns of plastic flow localization. In what follows the localized plastic flow patterns 
are discussed in the order of their emergence.

The first to appear is a solitary front of localized plastic flow, which travels along the specimen elongation axis at the yield plateau in the polycrystals $(n=0)$ or at the easy glide in the single crystals $(n \approx 0)$. In this case, an elastic-plastic transition takes place. The motion of such a front can be regarded as switching autowave [11]. The next pattern is a mobile set of parallel fronts separated by distance $\lambda$, which is observed at the linear work hardening stage in the single-crystal and polycrystalline specimens $(n=1 ; \sigma \sim \varepsilon)$. In accordance with [8], the latter pattern corresponds to a typical phase autowave having length $\lambda$ and propagation rate $V_{a w}$. The third in the order of appearance is a group of immobile equidistant localized plasticity nuclei, which occurs at the parabolic work hardening (Tailor's) stage $\left(n=1 / 2 ; \quad \sigma \sim \mathcal{E}^{1 / 2}\right)$ in most materials [9]. This pattern might be considered a stationary dissipative structure [11]. The autowave pattern emergent at the pre-failure stage $(0<n<1 / 2)$ is specifically associated with 'collapse' of the autowave [12] which takes place concurrently with the onset of macro-necking. The final stage of the plastic flow process $(n \approx 0)$ is ductile failure of material. The above phenomena would emerge spontaneously in the specimen under constant-rate tensile loading. Following Seeger and Frank [13], we regard these phenomena as processes of structure formation.

On the base of conclusive evidence obtained for a wide range of materials the following Rule of Correspondence is introduced: in accordance with the acting work hardening law, $\sigma(\varepsilon)$, each plastic flow stage involves a special kind of autowave process. The rule formulated above applies to all the plastic flow phenomena having a characteristic macro-scale of about $10^{-2} \mathrm{~m}$. However, the work hardening law governs the mechanisms involved in the interaction of lattice defects [6]. Therefore, the said rule apparently applies to the defect subsystem of the deforming crystal [9]. For this reason, the plastic flow is expected to manifest certain microscopic features that are indications of microscopic effects. In our opinion, three such manifestations merit special note. These are considered below.

\section{On the Manifestations of Microscopic Effects}

\subsection{Elastic and Plastic Deformation Invariant}

On the base of experimental data a significant regularity is established for the autowave process of plastic flow localization in a range of metals. Thus a close correlation is found to exist between the product of the macroscopic parameters of the autowave process, $\lambda \cdot V_{a w}$, and the product of the microscopic (lattice) parameters of material, $d \cdot V_{\perp}$. Here $d$ is the spacing between the close-packed planes of the lattice and $V_{\perp}$ is the transverse elastic wave rate. The numerical data obtained for studied metals is listed in Table 1. Matching of this data suggests that the following equality is good within an acceptable range of accuracy

$$
\lambda \cdot V_{a w} \approx 1 / 2 d \cdot V_{\perp} \approx 3 / 2 r_{i} \cdot V_{\perp}
$$

Indeed, a numerical analysis shows that $d \approx 3 r_{i}$ (here $r_{i}$ is the Pauling ion radius [14]). Equation (2) is validated by the fact that the average ratio $\left\langle 2 \lambda \cdot V_{a w} / d \cdot V_{\perp}\right\rangle$ $\approx 1.04 \pm 0.52$ obtained for studied metals is about close to unity and the dependence $\lambda / d\left(V_{\perp} / V_{a w}\right)$ is a linear one (see Figure 3).

Equation (2) is physically significant since it establishes a quantitative relationship between the micro-scale (lattice) characteristics ( $d$ and $V_{\perp}$ ) of elastic waves which govern elastic deformation processes on the one hand and the macro-scale characteristics ( $\lambda$ and $V_{a w}$ ) of localized plastic flow autowaves which are generated

Table 1. Matching of $\lambda \cdot V_{a w}$ and $d \cdot V_{\perp}$ values calculated from Equation (2).

\begin{tabular}{cccccc}
\hline \multirow{2}{*}{ Metal } & $\lambda \cdot V_{a w} \cdot 10^{7}$ & $d \cdot 10^{10}$ & $V_{\perp} \cdot 10^{-3}$ & $d \cdot V_{\perp} \cdot 10^{7}$ & \\
\cline { 2 - 4 } \cline { 2 - 4 } & $\left(\mathrm{m}^{2} \mathrm{~s}^{-1}\right)$ & $(\mathrm{m})$ & $\left(\mathrm{ms}^{-1}\right)$ & $\left(\mathrm{m}^{2} \mathrm{~s}^{-1}\right)$ & \\
\hline $\mathrm{Cu}$ & 3.60 & 2.08 & 2.30 & 4.78 & 1.50 \\
$\mathrm{Al}$ & 7.92 & 2.33 & 3.23 & 7.52 & 2.10 \\
$\mathrm{Zr}$ & 1.92 & 2.46 & 2.25 & 5.53 & 0.70 \\
$\mathrm{Ti}$ & 3.50 & 2.24 & 2.96 & 6.63 & 1.06 \\
$\mathrm{~V}$ & 2.80 & 2.14 & 2.83 & 6.06 & 0.92 \\
$\gamma-\mathrm{Fe}$ & 2.55 & 2.07 & 3.32 & 6.87 & 0.74 \\
$\alpha-\mathrm{Fe}$ & 2.24 & 2.03 & 3.32 & 6.74 & 0.66 \\
$\mathrm{Ni}$ & 2.10 & 2.03 & 3.22 & 6.54 & 0.64 \\
\hline
\end{tabular}

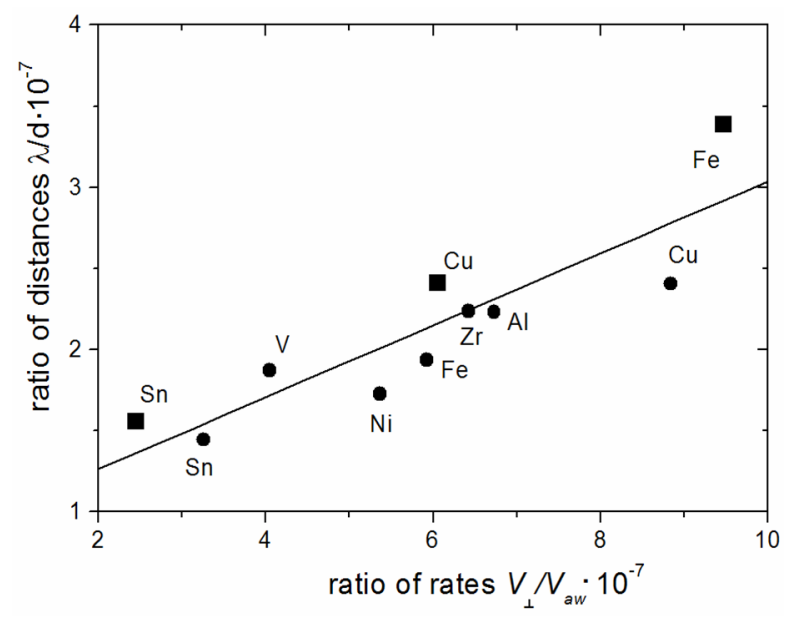

Figure 3. Verification of the validity of Equation (2) with help of a linear dependence between the ratios $\lambda / d$ and $V_{\perp} / V_{a w}$; - - easy glide stage; $\bullet$ - linear work hardening stage. 
in deforming media on the other hand. In this case, it might be reasonable to regard the products $d \cdot V_{\perp}$ and $\lambda \cdot V_{a w}$ as invariants of elastic and plastic deformation processes, respectively. The above regularity suggests that the elastic and the plastic processes simultaneously involved in the deformation ( $\varepsilon<<1$ and $\varepsilon \approx 1$, respectively) are closely related. The quantity $V_{\perp}$ is the rate of elastic stress redistribution in the deforming solid and the quantity $V_{a w}$ is the rate of localized plasticity front rearrangement in the same solid. Thus, the macro-scale localization of plastic deformation can no longer be regarded as a mere disturbance of plastic flow homogeneity by necking. What is more, the localization phenomena are taken to be an attribute of the plastic deformation, with their characteristics being closely associated with and largely determined by the properties of crystal lattice.

\subsection{Autowave Characteristics and the Planck Constant}

A numerical analysis suggests that for all metals and alloys studied the following equality holds good:

$$
\lambda \cdot V_{a w} \cdot \rho \cdot r_{i}^{3}=h,
$$

where $\rho$ is material density. In other words, the quantum (Planck's) constant $h$ can be calculated by multiplying the values $V_{a w}$ and which are localized plastic flow characteristics measured experimentally by the values and $r_{i}$ which are hand-book material constants. Indeed, the calculated values $h$ listed in Table 2 are close to the Planck constant $h=6.626 \cdot 10^{-34} \mathrm{~J} \cdot \mathrm{s}$, with the average value $\langle h\rangle$ being $(6.44 \pm 0.88) \cdot 10^{-34} \mathrm{~J} \cdot \mathrm{s}$ and the ratio $\langle h\rangle / h=0.96 \pm 0.07$ being close to unity. Thus the Planck constant can be estimated directly from the macro-scale characteristics $V_{a w}$ and $\lambda$, which appears striking in itself.

\subsection{Form of Dispersion Relation}

Let us consider the following quadratic dispersion law [15]

$$
\omega=\omega_{0} \pm \alpha\left(k-k_{0}\right)^{2},
$$

where $\alpha, \omega_{0}$ and $k_{0}$ are empirical constants. The values of these constants were derived for localized plastic flow autowaves from the experimental data in Figure 4. Note that the value $\alpha$ can be both negative and positive, i.e. for the easy glide stage, $\alpha<0$ and for the linear work hardening stage, $\alpha>0$. Equation (4) is readily reduced to the canonic form $\tilde{\omega}=1 \pm \tilde{k}^{2}$ by substituting $\omega=\omega_{0} \cdot \tilde{\omega}$ and $k=k_{0}+\frac{\tilde{k}}{\sqrt{\operatorname{sign} \alpha \cdot \alpha / \omega_{0}}}$ (here $\tilde{\omega}$ and $\tilde{k}$ are the di- mensionless frequency and the wave number, respectively, and the signum function of $\alpha$ is $\operatorname{sign} \alpha\left\{\begin{array}{l}=+1 \\ =-1\end{array}\right.$ for $\alpha>0$ for $\alpha<0$ ). Wave processes that have quadratic dispersion law of the latter form would generally satisfy a number of nonlinear equations, e.g. the Schrödinger nonlinear equation, the sine-Gordon equation, etc., which are frequently employed to address self-organization processes occurring in nonlinear media [16]. Therefore, the dispersion relation (4) is taken to be an additional proof for plastic flow localization being involved in the self-organization of the deforming medium. Moreover, Equation (4) turned out to be formally equivalent to the de Broglie wave dispersion law for electrons in sharpcornered potential well [17], which is significant in itself.

\section{A Postulate of Plastic Flow Localization and the Ensuing Corollaries: Introduction of a New Quasi-particle}

Taken as a whole the above localization patterns demonstrate that the phenomenon in question has not only macroscopic characteristics of autowave process ( $\lambda$ and $V_{a w}$ ) but also microscopic ones ( $h$ and $d$ or $r_{i}$ ). In view of the ratio $\lambda / r_{i}$ being about equal to $10^{8}$, the key problem in this case is how the macro- and micro-scales could be reconciled. To overcome this problem, we propose to use an approach based on the wave-particle dualism, which received wide application in the physics of solids [18]. We are led to postulate the existence of aquasi-particle having effective mass, $m_{\text {ef }}$, quasimomentum, $p$, and energy, E, which corresponds to the autowave of localized plastic deformation having wavelength, $\lambda$ and

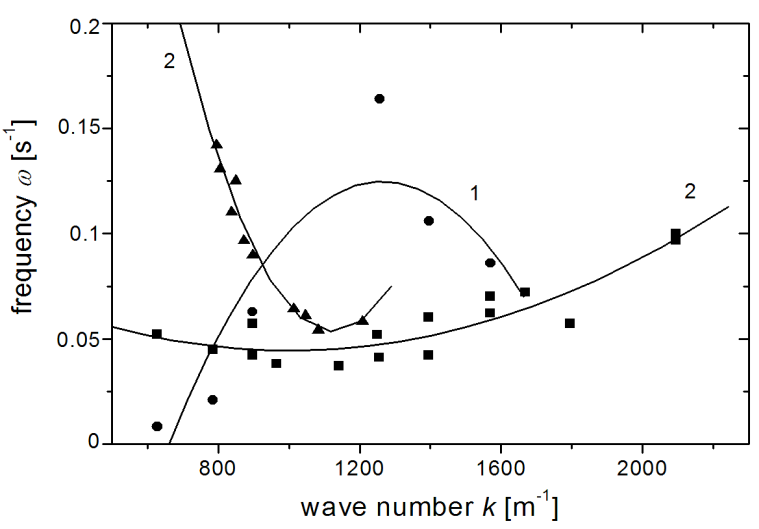

Figure 4. The dispersion law $\omega(k)$ established for localized plastic flow autowaves generated at the stages of easy glide (1) and linear work hardening (2); • - single crystals of $\mathrm{Cu}, \mathrm{Sn}$ and alloyed $\gamma$-Fe; $\square-$ single crystals of alloyed $\gamma$-Fe; $\Delta$ - polycrystalline Al. 
Table 2. Microscopic characteristics and the Planck constant values calculated from the data on localized plastic flow autowaves.

\begin{tabular}{|c|c|c|c|c|c|c|c|c|c|c|}
\hline \multirow[t]{2}{*}{ Metal } & $\lambda \cdot 10^{3}$ & $V_{a w} \cdot 10^{5}$ & $m_{e f}$ & $\mu \cdot 10^{2}$ & $n$ & $\Omega \cdot 10^{27}$ & $d_{\Omega} \cdot 10^{9}$ & $r_{\text {ion }} \cdot 10^{9}$ & \multirow[t]{2}{*}{$d_{\Omega} / r_{i o n}$} & \multirow{2}{*}{$\begin{array}{c}h \cdot 10^{34} \\
(\mathrm{~J} \cdot \mathrm{s})\end{array}$} \\
\hline & (m) & $\left(\mathrm{ms}^{-1}\right)$ & (a.m.u) & & & $\left(\mathrm{m}^{3}\right)$ & $(\mathrm{m})$ & (m) & & \\
\hline $\mathrm{Cu}$ & 4.5 & 8.0 & 1.1 & 1.74 & 1 & 0.21 & 0.059 & 0.072 & 0.82 & 8.14 \\
\hline $\mathrm{Al}$ & 7.2 & 11 & 0.50 & 1.87 & 3 & 0.31 & 0.068 & 0.051 & 1.33 & 5.0 \\
\hline $\mathrm{Zr}$ & 5.5 & 3.5 & 2.05 & 2.24 & 4 & 0.53 & 0.081 & 0.079 & 1.03 & 6.13 \\
\hline $\mathrm{Ti}$ & 7.0 & 5.0 & 1.1 & 2.3 & 4 & 4.2 & 0.075 & 0.076 & 0.99 & 6.91 \\
\hline $\mathrm{V}$ & 4.0 & 7.0 & 1.42 & 2.81 & 5 & 0.33 & 0.069 & 0.059 & 1.17 & 6.52 \\
\hline$\gamma-\mathrm{Fe}$ & 5.0 & 5.1 & 1.76 & 2.81 & 8 & 0.33 & 0.069 & 0.064 & 1.08 & 6.32 \\
\hline$\alpha-F e$ & 4.3 & 5.2 & 1.77 & 3.0 & 8 & 3.75 & 0.072 & 0.064 & 1.13 & 6.32 \\
\hline $\mathrm{Ni}$ & 3.5 & 6.0 & 0.89 & 3.24 & 10 & 0.32 & 0.068 & 0.069 & 0.99 & 6.17 \\
\hline
\end{tabular}

propagation velocity, $V_{a w}$. Then it can be written that

$$
m_{e f}=h / \lambda V_{a w} \text {. }
$$

Equality (5) is a mathematical expression of the above postulate to which we conventionally add the equations $p=m_{e f} V_{a w}=\hbar k$ and $E=\hbar \omega \quad$ (here $\hbar=h / 2 \pi$ ) for momentum and energy, respectively $[19,20]$. It is common knowledge that to validate a postulate, one has to match the corollaries ensuing from the same against experimental evidence. It turns out that a set of corollaries ensues from the above postulate which give an insight into the nature of localized plastic flow processes. These corollaries are considered below.

Corollary 1. First we will demonstrate that the effective mass, $m_{e f}$, calculated from Equation (5) has a physical meaning; this quantity depends on the characteristics of the deforming crystal. Indeed, the calculated values listed in Table 2 for a number of metals are in the range $0.5 \leq m_{e f} \leq 2$ a.m.u. (here 1 a.m.u. $=1.66 \cdot 10^{-27} \mathrm{~kg}$ is atomic mass unit). Evidently, the volume is readily calculated as $\Omega=m_{e f} / \rho$; then the length is found as $d_{\Omega}=\sqrt[3]{\Omega}$. The latter value is close to the value $r_{i}$, i.e. $d_{\Omega} \approx r_{i}$, with the average ratio being $\left\langle d_{\Omega} / r_{i}\right\rangle=1.07 \pm$ $0.09 \approx 1$. Thus, the effective mass, $m_{e f}$, turns out to be related to the lattice characteristics, $\rho$ and $r_{i}$.

Apparently, the values $m_{e f}$ calculated from Equation (5) differ slightly for each particular metal. The normalization of effective masses, $m_{e f}$, to the atomic masses, $A_{m}$, of respective metals yields normalized (dimensionless) mass $\mu=m_{e f} / A_{m}<<1$, which increases linearly with the number of electrons per unit cell, $n$ (see Figure 5) [21], i.e.

$$
\mu=\mu_{0}+\kappa \cdot n
$$

The correlation coefficient for $\mu$ and $n$ values is $\sim 0.95$; it has a high statistical significance.

Now we propose a physical interpretation of Equation (6) which is based on the consideration of jump-like dislocation motion. Thus a dislocation would become ar-

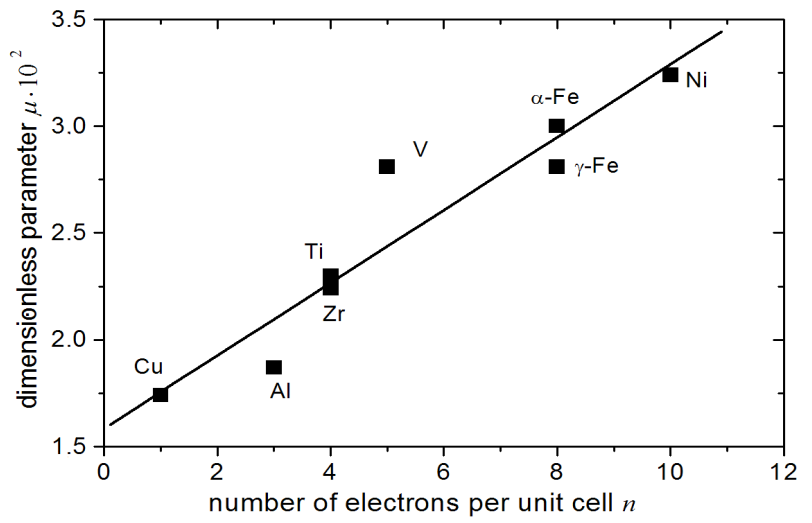

Figure 5. The dimensionless parameter, as a function of the number of electrons per unit cell, $n$.

rested at a local barrier until the thermal fluctuation causes its breakaway, which suggests that the plastic deformation results from the dislocation motion in a viscous medium [22]. For $V_{\text {disl }}=$ const, the motion of dislocations is controlled by viscous drag force per unit length, $F_{v} \approx B V_{\text {disl }}$ (here $B$ is the viscous drag factor for dislocations) [22]. For $V_{\text {disl }} \neq$ const, an inertial term proportional to the dislocation acceleration, $\dot{V}_{d i s l}$, is added to the viscous drag force [23]. Then the total drag force, $F_{\Sigma}$, is given by

$$
F_{\Sigma}=F_{v}+F_{i n} \approx B V_{d i s l}+\left(B / \omega_{a}\right) \cdot \dot{V}_{d i s l},
$$

where $\omega_{a}$ is the frequency of an elementary deformation act and $B / \omega$ apparently has the meaning of added mass per unit length of dislocation.

In the case of metals, the factor $B$ is determined by the interaction of dislocations with phonon and electron gases [22]; moreover, the contributions of phonon and electron gases, i.e. $B_{p h}$ and $B_{e}$, respectively, are additive so that $B=B_{p h}+B_{e}$. In this case, the first and the second term in the right-hand side of Equation (6) are evidently connected with the contributions to the added mass, $B / \omega$, of the viscous drag of both gases. 
The term $\mu_{0}$ from (6) is apparently independent of the kind of metal, which is reasonable since the properties of metal are only weakly dependent on the characteristics of its phonon spectrum at temperatures exceeding the Debye temperature [21]. However, the contribution of electron gas to the added mass should be proportional to $n$, i.e. $B_{e} \sim n$ [22]. Hence it can be written

$$
F_{i n} \sim\left(B_{p h}+B_{e}\right) / \omega \cdot \dot{V}_{d i s l} \sim\left(m_{p h}+m_{e}\right) \cdot \dot{V}_{d i s l} \sim\left(\mu_{0}+\kappa \cdot n\right) \cdot \dot{V}_{d i s l}
$$

Thus Equation (8) relates the normalized mass, $\mu$, to the contributions of phonon and electron gases.

Corollary 2. Equation (5) can be rewritten as

$$
\lambda V_{a w}=\frac{h}{m_{e f}}=\frac{h}{\rho \cdot r_{i}^{3}}=\varsigma \text {. }
$$

The quantity $\varsigma$ from (9) is calculated as $\varsigma=h / \rho r_{i}^{3}$. Then the calculated values, $\varsigma$, are matched with the experimental $\lambda V_{a w}$ data. The average values obtained for the single $\gamma$-Fe crystals and polycrystalline Al are, respectively, $\left\langle\lambda V_{a w}\right\rangle=(2.86 \pm 0.44) \cdot 10^{-7} \mathrm{~m}^{2} \cdot \mathrm{s}^{-1} \quad(\varsigma=3.2$. $\left.10^{-7} \mathrm{~m}^{2} \cdot \mathrm{s}^{-1}\right)$ and $\left\langle\lambda V_{a w}\right\rangle=(7.75 \pm 1.36) \cdot 10^{-7} \mathrm{~m}^{2} \cdot \mathrm{s}^{-1}(\varsigma=$ $\left.18.4 \cdot 10^{-7} \mathrm{~m}^{2} \cdot \mathrm{s}^{-1}\right)$.

Corollary 3. Equation (5) can be also rewritten as

$$
V_{a w}=\frac{h}{m_{e f} \cdot \lambda}=\frac{h}{\rho \cdot r_{i}} \cdot \frac{1}{\lambda}=\frac{\varsigma}{2 \pi} \cdot k \text {. }
$$

It is shown above that $V_{a w}=V_{g r}=d \omega / d k$ and $d \omega=(\varsigma / 2 \pi) \cdot k \cdot d k$. Hence we can write

$$
\int_{\omega_{0}}^{\omega} d \omega=\frac{\varsigma}{2 \pi} \int_{0}^{k-k_{0}} k \cdot d k .
$$

It follows from (11) that dispersion relation of quadratic form can be written for localized plastic flow autowaves, i.e. $\omega=\omega_{0}+\frac{\varsigma}{4 \pi}\left(k-k_{0}\right)^{2}=\omega_{0}+\alpha\left(k-k_{0}\right)^{2}$. Apparently, the latter relation corresponds to (4). The coefficient $\alpha$ from the dispersion relation of quadratic form can be found by matching the experimental $\omega(k)$ data against the calculated values $\alpha=\varsigma / 4 \pi$ obtained for $\mathrm{Fe}$ and $\mathrm{Al}$. Thus the experimental values $\alpha$ obtained for $\mathrm{Fe}$ and $\mathrm{Al}$ are $5.4 \cdot 10^{-8} \mathrm{~m}^{2} \cdot \mathrm{s}^{-1}$ and $7.9 \cdot 10^{-7} \mathrm{~m}^{2} \cdot \mathrm{s}^{-1}$, respectively, and the calculated data are $2.5 \cdot 10^{-8} \mathrm{~m}^{2} \cdot \mathrm{s}^{-1}$ and $1.46 \cdot 10^{-7} \mathrm{~m}^{2} \cdot \mathrm{s}^{-1}$, respectively. Both sets of data have practically the same order of magnitude.

Corollary 4. It follows from Equation (5) that

$$
\lambda V_{a w} \rho=h / r_{i}^{3} \text {. }
$$

The terms in both sides of (12) evidently have the units of dynamic viscosity, i.e. $\mathrm{kg} \cdot \mathrm{m}^{-1} \cdot \mathrm{s}^{-1} \equiv \mathrm{Pa} \cdot \mathrm{s}$. The calculated value $\lambda \cdot V_{a w} \cdot \rho$ is about equal to $5 \cdot 10^{-4} \mathrm{~Pa} \cdot \mathrm{s}$ for all studied metals. Hence the latter quantity can be identified with the viscosity of phonon gas, $B$, which controls dislocation mobility by quasi-viscous motion [22]. This is good indirect evidence for the validity of the same quantity, in particular, by interpreting the physical meaning of dependence (6).

Corollary 5. The dispersion relation obtained for localized plastic deformation autowaves can be rewritten in the form appropriate for quasi-particles, i.e. $E=E(p)=$ $E_{0}+\alpha^{\prime}\left(p-p_{0}\right)^{2}$ (here $E_{0}, p_{0}$ and $\alpha^{\prime}$ are constants). Then the effective mass of the hypothetical quasi-particle is estimated [17] as

$$
m_{e f}=\left(\partial^{2} E / \partial p^{2}\right)^{-1}=\hbar \cdot\left(\partial^{2} \omega / \partial k^{2}\right)^{-1} .
$$

The experimental and calculated effective masses obtained for iron and aluminum are 0.6 and 0.1 a.m.u., respectively. This lends credence to the existence of the hypothetical quasi-particle having $m_{e f} \approx 1$ a.m.u.

Corollary 6. As is seen from Figure 4, the oscillation spectrum $\omega=\omega_{0}+\alpha\left(k-k_{0}\right)^{2}$ has a narrow gap in the range $0 \leq \omega \leq \omega_{0} \approx 10^{-2} \mathrm{~Hz}$. Hence for any temperature, $\hbar \omega_{0}<<k_{B} T$ (here $k_{B}$ is the Boltzmann constant). Localization phenomena are liable to occur spontaneously at any temperature provided geometric constraints place no restrictions, in particular, in the case of small-sized specimens [1].

Corollary 7. Finally, the jump-like plastic deformation in solids [24] can be explained with help of the above postulate. Let us rewrite Equation (5) as

$$
\delta L \approx h\left(\rho r_{i} V_{a w}\right)^{-1} .
$$

Now suppose that the specimen length $L$ accommodates an integer $m=1,2,3 \ldots$ of autowaves having length $\lambda$, i.e. $\lambda=L / m$, which precludes the occurrence of deformation within the clamps of the testing machine. With growing total deformation, $\varepsilon$, the elongation of the specimen occurs as $L \approx L_{0}(1+\varepsilon) \approx L_{0}+\delta L$ (here $L_{0}$ is the original specimen length). Hence from (14) follows that

$$
\delta L \approx h\left(\rho r_{i} V_{a w}\right)^{-1} \cdot m .
$$

For the linear work hardening stage, $V_{a w}=$ const . From (15) apparently follows that the specimen length would vary discretely $(\delta L \sim m)$ in accord with the jump-wise deformation behavior, i.e. the specimen length would be accommodated to the emergent autowave pattern. Deformation jumps may occur by different mechanisms depending on the kind of material so that Equation (15) only states that this kind of deformation behavior is a must.

From (15) follows that $\delta L \sim V_{a w}^{-1}$. The available experimental evidence [1] suggests that the autowave 
propagation rate is proportional to the cross-head motion velocity, i.e. $V_{a w} \sim V_{\text {mach }}$. With increasing velocity of the movable clamp of the test machine, the amplitude of jumps is expected to grow less. The latter inference is supported by the experimental data obtained for $\mathrm{Al}$ at 1.4 $\mathrm{K}$ [24].

Corollary 8. Now it transpires that Equation (3) of the form $\lambda \cdot V_{a w} \cdot \rho \cdot r_{i}^{3}=h$ is readily derived from the same postulate provided $m_{e f}=\rho \cdot r_{i}^{3}$.

\section{Conclusions}

It is pertinent to mention here the early efforts to apply quantum ideas to problems of strength and plasticity physics. Thus Steverding was the first to introduce the notion of elastic waves quantization by fracture [25]. By addressing the kinetics of brittle fracture, the existence of a quasi-particle in the deforming medium was postulated. The quasi-particle was identified with the tip of a growing crack; therefore, it got the name 'crackon' [26]. Quantum models were applied to explain the tunnel effect observed by dislocation motion [27,28]. On the other hand, recent theoretical and experimental studies indicate that the plastic flow involves wave processes $[1-5,7,29$, 30].

The above findings justify the use of quantum concepts to address plastic flow development in solids. In this approach one has to reconcile micro- and macroscale manifestations of the localization phenomenon; therefore, physicists dealing with plasticity problems might consider it invalid. When the micro-scale (dislocation) level is addressed, the quantization idea appears quite appropriate. In view of crystal lattice discreteness, the minimal possible shear is by the Burgers vector, $b$, which has microscopic scale $d$ of about $10^{-10} \mathrm{~m}$ and hence might be regarded as a 'quantum of shear deformation'. Hence the use of (2) allows the quantization concept to be extended to the macro-scale level, i.e. $\lambda \cdot V_{a w} \approx 1 / 2 d \cdot V_{\perp}$.

The fact that the Planck constant value calculated from the data derived in rather 'rough' macro-experiments is close to a handbook value might be due to the manifestation of so-called 'universality concept' [31], which postulates that the system's measurables are virtually unaffected by the distribution of its major microscopic properties. In other words, both the qualitative and the quantitative characteristics of material substructure are only partly responsible for, e.g. plastic flow evolution, flow stress level, work hardening coefficient, etc.

The corollaries ensuing from the postulate proposed herein have proven to be very fruitful to study deformation localization processes, which validates the concept of wave-particle duality. By way of summary it should be emphasized that the macro-scale effects (characteris- tic scale $\mathrm{L}_{\text {macro }} \approx \lambda$ ) emerging in a plastically deforming multi-scale system are found to be directly related to the micro-scale effects (characteristic scale $\mathrm{L}_{\text {micro }} \approx r_{i}$ ), with the scale ratio being $\mathrm{L}_{\text {macro }} / \mathrm{L}_{\text {micro }} \approx \lambda / r_{i} \geq 10^{8}$.

The above gives justification to the use of a common approach, i.e. postulation of a quasi-particle corresponding to a localized plastic flow autowave. This turns out to be a fruitful effort by explaining a number of relationships in plasticity physics, which have remained poorly understood in the frame of traditional models of crystal plasticity. The postulated quasi-particle would be named "auto-localizon".

As far back as the 1960-ies Dzyaloshinski [32] was the first to discuss the possibility of measuring the Planck constant in mechanical experiments. In his paper this worker emphasizes the importance of studying the macroscopic manifestations of typical quantum effects, e.g. superfluidity, superconductivity and the quantum Hall effect discovered later on. In the light of the foregoing it is maintained that the plastic flow in solids is analogous to all these phenomena; therefore, this might also be regarded as a macroscopic quantum effect.

\section{Acknowledgements}

This work was partly supported by the grant of RFBR (09-08-00213-a).

\section{References}

[1] L. B. Zuev, "Wave Phenomena in Low-Rate Plastic Flow of Solids," Annals of Physics, Vol. 10, 2001, pp. 965-984.

[2] L. B. Zuev, "On the Waves of Plastic Flow Localization in Pure Metals and Alloys," Annals of Physics, Vol. 16, 2007, pp. 286-310.

[3] R. J. McDonald, C. Efstathiou and P. Curath, "The Wave-Like Plastic Deformation of Single Crystals Copper," Journal of Engineering Materials and Technology, Vol. 131, 2009, pp. 692-703.

[4] A. Asharia, A. Beaudoin and R. Miller, "New Perspectives in Plasticity Theory: Dislocation Nucleation, Waves and Partial Continuity of Plastic Strain Rate," Mathematics and Mechanics of Solids, Vol. 13, 2008, pp. 292-315.

[5] C. Fressengeas, A. Beaudoin and D. Entemeyer, "Dislocation Transport and Intermittency in the Plasticity of Crystalline Solids," Physical Review B, Vol. 79, 2009, pp. 014108-1-014108-9.

[6] L. B. Zuev, V. V. Gorbatenko and S. N. Polyakov, "Instrumentation for Speckle Interferometry and Techniques for Investigating Deformation and Fracture," Proceedings of SPIE, Vol. 4900, Part 2, 2002, pp. 1197-1208.

[7] H. Haken, "Information and Self-Organization," A Macroscopic Approach to Complex Systems, Springer Verlag, Berlin, 1989.

[8] H. Kolsky, "Stress Waves in Solid," Phoenix Education, 
Dover, 2003.

[9] F. R. N. Nabarro, Z. S. Basinski and D. B. Holt, "The Plasticity of Single Crystals," Tailor and Francis Ltd, London, 1964.

[10] R. Hill, "The Mathematical Theory of Plasticity," University Press, Oxford, 1998.

[11] V. A. Davydov, V. S. Zykov and A. S. Michailov, "Kinematics of Autowave Structures in the Excited Media," Physics-Uspekhi, Vol. 161, 1999, pp. 45-85.

[12] B. B. Kadomtsev, "Dynamics and Information," PhysicsUspekhi, Vol. 164, 1994, pp. 449-530.

[13] A. Seeger and W. Frank, "Structure Formation by Dissipative Processes in Crystals with High Defect Densities," In L. P. Kubin and G. Martin Eds., "Non-Linear Phenomena in Material Science," Trans Tech Publications, New York, 1987, pp. 125-137.

[14] L. Pauling, "The Nature of the Chemical Bond," Cornell University Press, Ithaca, 1960.

[15] S. A. Barannikova, "Dispersion of the Plastic Strain Localization Waves," Technical Physics Letters, Vol. 30, 2004, pp. 338-340.

[16] A. Scott, "Nonlinear Science," Emergence and Dynamics of Coherent Structures, University Press, Oxford, 2003.

[17] L. D. Landau and E. M. Lifschitz, "Quantum Mechanics: Non-Relativistic Theory," Course of Theoretical Physics, Pergamon, London, 1977.

[18] J. M. Ziman, "Electrons and Phonons," University Press, Oxford, 2001.

[19] J. P. Billingsley, "The Possible Influence of the De Broglie Momentum-Wavelength Relation on Plastic Strain 'Autowave' Phenomena in 'Active Materials'," International Journal of Solids and Structures, Vol. 38, 2001, pp. 4221-4234.

[20] L. B. Zuev, "The Linear Work Hardening Stage and De Broglie Equation for Autowaves of Localized Plasticity," International Journal of Solids and Structures, Vol. 42, 2005, pp. 943-949.
[21] A. P. Cracknell and K. C. Wong, "The Fermi Surface. Its Concept, Determination and Use in the Physics of Metals," Clarendon Press, Oxford, 1973.

[22] V. I. Al'shits and V. L. Indenbom, "Mechanisms of Dislocations Drag," In F. R. N. Nabarro Ed., Dislocations in Solids, Amsterdam, 1986, pp. 43-111.

[23] L. D. Landau and E. M. Lifshits, "Hydrodynamics," Nauka Publications, Moscow, 1988.

[24] V. V. Pustovalov, "Serrated Deformation of Metals and Alloys at Low Temperatures," Low Temperature Physics, Vol. 34, 2008, pp. 683-723.

[25] B. Steverding, "Quantization of stress waves and Fracture," Materials Science and Engineering, Vol. 9, 1972, pp. 185-189.

[26] E. M. Morozov, L. S. Polack and Ya. B. Fridman, "On Variation Principles of Crack Development in Solids," Soviet Physics-Doklady, Vol. 156, 1964, pp. 537-540.

[27] T. Oku and J. M. Galligan, "Quantum Mechanical Tunneling of Dislocations," Physical Review Letters, Vol. 22, 1969, pp. 596-597.

[28] B. V. Petukhov and V. L. Pokrovsky, "Quantum and Classic Motion of Dislocations in the Potential Peierls Relief," Journal of Experimental and Theoretical Physics, Vol. 63, 1972, pp. 634-647.

[29] E.C. Aifantis, "Nonlinearity, Periodicity and Patterning in Plasticity and Fracture," International Journal of NonLinear Mechanics, Vol. 31, 1996, pp. 797-809.

[30] E.C. Aifantis, "Gradient Plasticity," In J. Lemaître, Ed., Handbook of Materials Behavior Models, Academic Press, New York, 2001, pp. 291-307.

[31] Y. Imry, "Introduction to Mesoscopic Physics," University Press, Oxford, 2002.

[32] L. P. Dzyaloshinski, "Macroscopic Quantum Phenomena," Soviet Physics-Uspekhi, Vol. 90, 1966, pp. 623629. 